\title{
Особенности характеристик устойчивых к полевым повреждениям структур кремний-сверхтонкий окисел-поликремний
}

\author{
(C) Е.И. Гольдман, С.А Левашов, Г.В. Чучева \\ Фрязинский фрилиал Института радиотехники и электроники им. В.А. Котельникова \\ Российской академии наук, \\ 141190 Фрязино, Россия \\ E-mail: gvc@ms.ire.rssi.ru
}

(Получена 23 октября 2018 г.

Принята к печати 26 октября 2018 г.

Принята к публикации 29 октября 2018 г.)

\begin{abstract}
Приведены результаты исследований особенностей вольт-амперных и вольт-фарадных характеристик структур кремний-сверхтонкий окисел-поликремний, устойчивых к полевым повреждениям. Оказалось, что происходящая с изменением полевого напряжения общая перезарядка сконцентрированных у границы раздела подложка-изолятор локализованных электронных состояний и неосновных носителей на порядок выше, чем у подверженных повреждению полевым стрессовым воздействием образцов. Туннельная вольт-амперная характеристика имеет существенно асимметричный вид: ток, протекающий из полевого электрода в кремниевую подложку, на несколько порядков меньше тока, протекающего из кремния в поликремний, при одинаковых значениях внешнего напряжения, падающего на изолирующий слой. Для объяснения такой асимметрии предположено, что в переходном слое от поликремния к окислу потенциальный барьер, разделяющий полупроводниковые электрод и подложку, имеет высоту $\sim 1$ эВ и поэтому всегда препятствует электропереносу; для обратных потоков этот барьер перестает ограничивать проводимость, как только уровень туннелирования станет выше него.
\end{abstract}

DOI: 10.21883/FTP.2019.04.47444.9011

Электрические поля, возникающие при управляющих воздействиях в сверхтонких изолирующих слоях наномасштабных активных элементов современных полупроводниковых устройств, достигают нескольких единиц $10^{6} \mathrm{~B} /$ см. Действие на структуры $n-\mathrm{Si}-\mathrm{SiO}_{2}$ такого сильного, хотя и допробойного, электрического поля, порождает в пленках окисла кремния несколько идущих с разной интенсивностью процессов. Во-первых, это захват электронов из полупроводника и затвора на развернутые вдоль электрических силовых линий связи $\mathrm{Si}-\mathrm{O}$. В результате существенно снижается энергия активации для термического отрыва отрицательно заряженного атома кислорода от кремния и тем самым образования в окисле кислородных вакансий. В настоящее время считается, что накопление со временем данных вакансий и соединение их в линии, закорачивающие управляющий электрод и полупроводниковую базу, являются основным механизмом развития пробоя изолирующего слоя структур металл-окисел-полупроводник (МОП) [1-3]. Во-вторых, происходят перемещение присутствующего в $\mathrm{SiO}_{2}$ водорода к поверхностям окисла, его реакции с пассивированными и не пассивированными оборванными связями на границах раздела (ГР) $\mathrm{Si}-\mathrm{SiO}_{2}$ и увеличение в связи с этим концентрации поверхностных электронных ловушек, так называемых $P_{b}$-центров [4-6]. В-третьих, вблизи ГР $\mathrm{Si}-\mathrm{SiO}_{2}$ происходит накопление встроенного заряда, который связан как с перераспределением ионов по окислу, так и с перезарядкой имеющихся и родившихся при полевом воздействии локализованных электронных состояний. Перечисленные явления сопровождаются повышением проводимости через изолирующий слой как за счет формирования новых каналов - образования коротких (не сквозных) линий из вакансий кислорода и повышения роли туннелирования через электронные уровни в окисле (trap-assisted current), так и в связи с дополнительным электрическим полем из-за накопления встроенного заряда [2]. $\mathrm{C}$ точки зрения изменения свойств контакта $\mathrm{Si}-\mathrm{SiO}_{2}$ последствия упомянутых процессов можно разделить на обратимые, так называемое „повреждение окисла“, и на необратимые, мягкий и жесткий пробой (soft breakdown or breakdown) изолирующего слоя. Подчеркнем, что, несмотря на допробойные значения рассматриваемых электрических полей, с ростом продолжительности воздействия возникает вероятность перехода образца в необратимое состояние мягкого пробоя [2,3].

Несколько лет нашей группой изучаются изменения свойств сверхтонких (<5нм) окислов кремния после повреждения МОП структур в электрических полях [7-9]. Выяснилось, что имеется группа образцов, в которых реакция на полевое воздействие типа „повреждение“ практически отсутствует, т. е. с увеличением продолжительности выдержки при полевых напряжениях разной полярности вплоть до перехода в состояние мягкого пробоя практически не изменяются ни проводимость через окисел, ни распределение встроенного заряда. В данной работе будут рассмотрены особенности характеристик таких объектов. Параметры исследованных образцов те же, что и параметры объектов, в которых наблюдалось явление „повреждения“ $[7,8]$ : это МОП струк- 
туры на $\mathrm{Si}$ с полевым электродом $\mathrm{Al}-n^{+}-\mathrm{Si}: \mathrm{P}$ (концентрация доноров в поликремнии $N_{d}^{+} \approx 10^{20} \mathrm{~cm}^{-3}$, площадь $\left.S=1.6 \cdot 10^{-3} \mathrm{~cm}^{2}\right)$, изолированным от подложки (100) $n$-Si слоем пирогенного окисла с оптической толщиной $\sim 4$ нм. Структуры подвергались полевому стрессовому воздействию при комнатной температуре при таких же полевых напряжениях $V_{g}$, как и в случае исследования повреждения сверхтонкого $\mathrm{SiO}_{2}[7,8]: V_{g}=-3.8 \mathrm{~B}$ (обеднение подложки), минимальное время выдержки 16 мин и $V_{g}=3.2$ В (обогащение подложки), минимальное время выдержки 20 мин. До и после стрессового воздействия проводились измерения вольт-амперной (BАХ) и высокочастотных вольт-фарадных характеристик (ВФХ) объектов на частотах 1 и $0.5 \mathrm{MГц} \mathrm{(емкости} C_{1}$ и $\left.C_{2}\right)$ с использованием прецизионного измерителя LCR Agilent E4980A [9]. Чтобы значения высокочастотных емкостей и тока отвечали одному и тому же состоянию образца, опыты проводились следующим образом: для каждой точки измерений из положения $V_{g}=0$ на образец подавалось заданное полевое напряжение; после окончания $R C$-процессов (времена $<0.1 \mathrm{c}$ ) регистрировался ток, затем в течение $3 \mathrm{c}$ емкости $C_{1}$ и $C_{2}$. После этого поданное напряжение $V_{g}$ сбрасывалось до нуля и структура выдерживалась 6 с. Общая продолжительность измерений тока и емкостей при одном и том же значении напряжения на полевом электроде (6 c) существенно меньше, чем характерное время переходного процесса, связанного с перезарядкой локализованных электронных состояний на ГР $\mathrm{Si}-\mathrm{SiO}_{2}$ (> $100 \mathrm{c}$ ). Такая процедура позволяет при измерениях минимизировать длительность пребывания образца в стрессовых условиях. Данные ВФХ, соответствующие двум высоким частотам, дают возможность определять концентрацию доноров вблизи ГР $\mathrm{Si}-\mathrm{SiO}_{2} N_{d}$, сопротивление полупроводниковой подложки $R_{b}$ и зависимости от полевого напряжения следующих величин: изгиба зон в полупроводнике $V_{s}$, падения внешнего напряжения на изолирующем слое $V_{i}$ и выраженной в см$^{-2}$ суммарной плотности встроенного заряда, заряда пограничных состояний и дырок на ГР $p_{s q}[8,10]$.

На рис. 1 представлены полевые зависимости емкости $C\left(V_{g}\right)$ образца, измеренные в состоянии до полевого стрессового воздействия при комнатной температуре на двух частотах. Их изменения после полевых воздействий разных полярностей и продолжительностей выдержки не превышали $2 \%$, в отличие от объектов в [8], где после полевого воздействия фиксировалось уменьшение емкостей в разы. Аналогичная картина наблюдалась и в отношении токов сквозь изолирующий слой: после выдержки образцов в сильном поле ВАХ сдвигались не более чем на сотые доли вольта. В результате обработки данных ВФХ по процедуре, описанной в $[8,10]$, получилось: $N_{d}=1.14 \cdot 10^{15} \mathrm{~cm}^{-3}$, $R_{b}=88$ Ом; эти величины очень близки к значениям, определенным в [8]. Зависимость $p_{s q}\left(V_{g}\right)$ приведена на рис. 2. Видно, что масштаб перезарядки ГР $\mathrm{Si}-\mathrm{SiO}_{2}$ в данных объектах на порядок выше, чем в повреждаю-

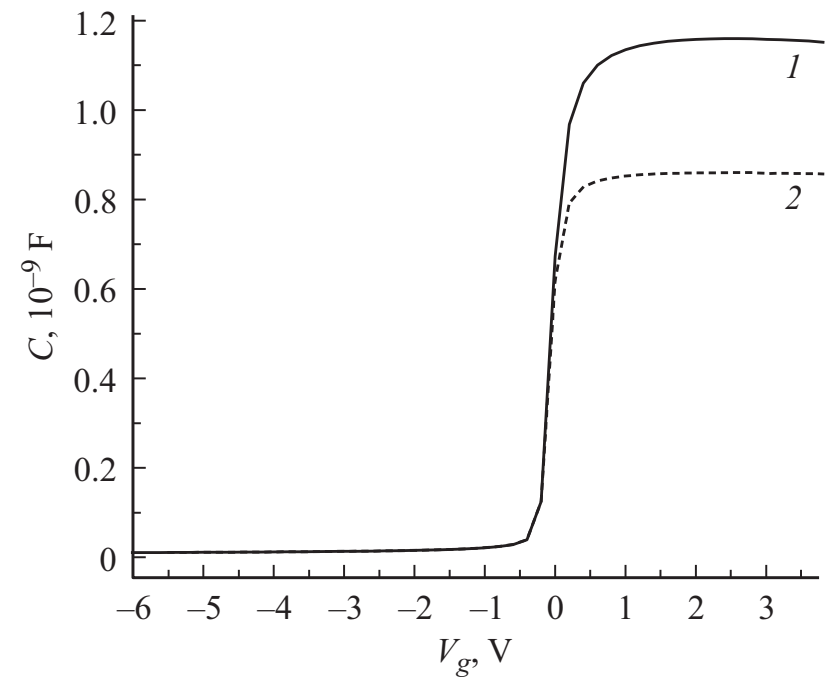

Рис. 1. Высокочастотные вольт-фарадные характеристики $C\left(V_{g}\right)$ кремниевой МОП структуры при частотах 0.5 (1) и $1 \mathrm{M} Ц$ (2).

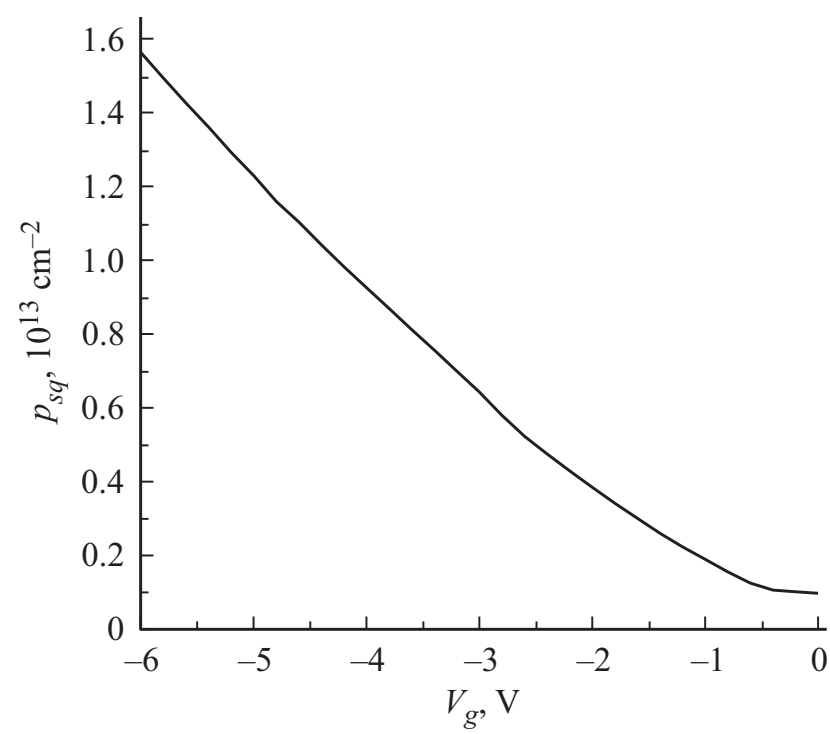

Рис. 2. Зависимость от полевого напряжения $V_{g}$ суммарной плотности встроенного заряда, заряда пограничных состояний и дырок на контакте подложка-окисел $p_{s q}$.

щихся структурах $[8,11]$ : при изменении $V_{g}$ от $-0,5$ до $-6 \mathrm{~B}$ увеличение $p_{s q}$ составило $\Delta p_{s q}=1.4 \cdot 10^{13} \mathrm{~cm}^{-2}$. При обедняющем полупроводник полевом напряжении оказалось, что $V_{i} \propto V_{g} / 2$. Это означает, что для данных $V_{g}$ образец находился в нестационарном состоянии и неравновесном по отношению к неосновным носителям заряда (генерация дырок запаздывает). На рис. 3 показаны логарифмические зависимости ветвей тока через окисел от падения внешнего напряжения на изолирующем слое, $\varphi_{ \pm}(V)$. Здесь $V=V_{g}$ при $V_{g}>0$ и $V=-V_{i}$ при $V_{g}<0$; для $V_{g}>0 \quad \varphi_{+}(V)=\lg \left[I\left(V_{g}\right) /\left.I\right|_{V_{g}=1 \mathrm{~V}}\right]$, для $V_{g}<0 \quad \varphi_{-}(V)=\lg \left[I\left(V_{i}\right) /\left.I\right|_{V_{i}=-1 \mathrm{~V}}\right], \quad I-$ ток в цепи 


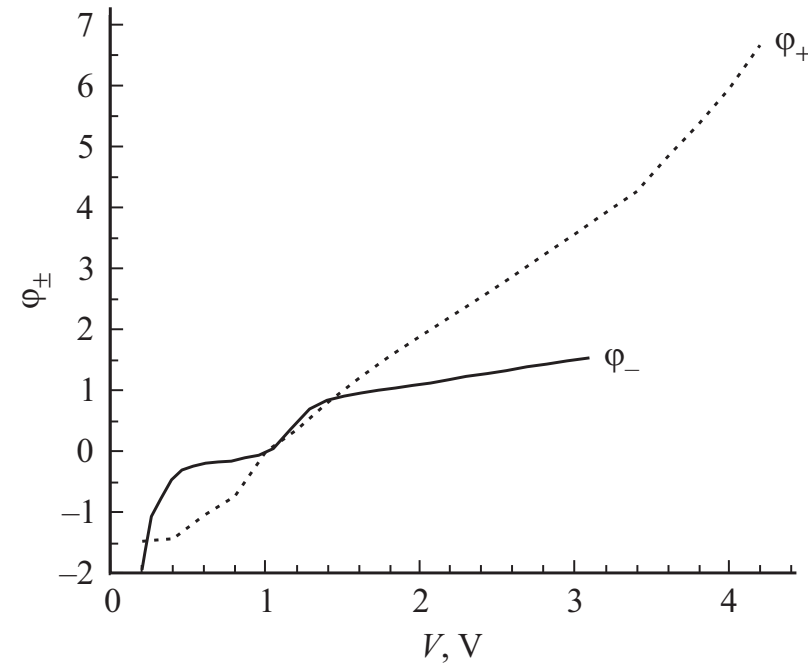

Рис. 3. Логарифмический вид туннельных вольт-амперных характеристик изолирующего слоя кремниевой МОП структуры. Для $V_{g}>0 \quad \varphi_{+}(V)=\lg \left[I\left(V_{g}\right) /\left.I\right|_{V_{g}=1 \mathrm{~V}}\right], \quad$ для $V_{g}<0$ $\varphi_{-}(V)=\lg \left[I\left(V_{i}\right) /\left.I\right|_{V_{i}=-1 \mathrm{~V}}\right]$.

полевой электрод-подложка. Нормировки тока $\left.I\right|_{V_{g}=1 \mathrm{~V}}$ и $\left.I\right|_{V_{i}=-1 \mathrm{~V}}$ выбрали исходя из того, что данные значения напряжений и токов по модулю примерно одинаковы: $\left.I\right|_{V_{g}=1 \mathrm{~V}}=5.6 \cdot 10^{-12} \mathrm{~A},\left.I\right|_{V_{i}=-1 \mathrm{~V}}=-4.8 \cdot 10^{-12} \mathrm{~A}$. Представленные на рис. 3 ВАХ отвечают туннельному переносу через изолирующий барьер: для проверки этого утверждения при нескольких напряжениях $V_{g}$ были измерены временны́е зависимости тока и емкостей на частотах 1 и 0.5 МГц. Вычисленные на этой основе токи перезарядки $q S\left(d p_{s q} / d t\right)(q-$ элементарный заряд, $t$ - время) по абсолютному значению оказались на 1.5-2 порядка меньше $I$. Из рис. 3 следует, что имеет место сильная асимметрия туннельной ВАХ по отношению к полярности падающего на изоляторе напряжения. Если для напряжений $V<1$ характеристики $\varphi_{+}(V)$ и $\varphi_{-}(V)$ примерно одинаковы, то при $V>1$ крутизна нарастания тока при положительных напряжениях на 2-3 порядка выше, чем при отрицательных: при 3 В на окисле $I=2 \cdot 10^{-8} \mathrm{~A}$, а при $-3 \mathrm{~B} I=-1.6 \cdot 10^{-10} \mathrm{~A}$. Такое поведение BAX не должно быть типичным для кремниевых МОП структур со сверхтонким окислом, где в условиях прямого туннелирования несимметрия может быть связана с несовпадением энергий Ферми в полупроводниках по разные стороны от изолятора, и поэтому должна быть относительно слабо выражена. Экспериментальные зависимости $I\left(V_{g}\right)$, представленные на рис. 15.12 обзора [2], подтверждают необычность кривых, приведенных на рис. 3. Отметим, что описанная в данной работе асимметрия $\mathrm{BAX}$, соответствующих разной полярности полевого напряжения, совершенно иная, чем рассмотренная в [12]. В указанной статье анализировалась ситуация, когда при малых $V_{g}$ ток, протекающий из полевого электрода в полупроводник, был меньше тока, протекающего из кремния в полевой электрод, но с ростом напряжения данный эффект исчезал.

Переходные слои между кристаллическим кремнием и его окислом занимают не менее 40\% объема сверхтонкого диэлектрика [13]. Фактически данные слои являются важными составляющими структуры окисла, во многом определяющими его свойства. Поэтому потенциальный барьер изолирующего промежутка имеет гораздо более сложную форму, чем прямоугольная [14]. Поскольку образование переходов от подложки к окислу и от окисла к поликремнию происходит в разных технологических процессах, следует ожидать и разных (не симметричных) по форме зависимостей потенциала от координаты на этих контактах. Это означает, что переход от $\mathrm{SiO}_{2}$ к поликремнию должен быть более широким, чем со стороны кремниевой подложки. В этой связи добавим, что на форму координатной зависимости потенциала должна влиять и поликристалличность полевого электрода. Для объяснения описанной выше асимметрии туннельной ВАХ кремниевых МОП диодов достаточно предположить, что структура потенциала в изоляторе содержит в переходном слое со стороны поликремния растущую с удалением от полупроводника ветвь, переходящую на высоте $\sim 1$ эВ в основной высокий барьер, простирающийся до контакта с кремниевой подложкой. Такая ветвь в дополнение к основному барьеру будет всегда ограничивать электроперенос из полевого электрода в подложку, а для обратного потока она перестанет влиять на проводимость, как только уровень туннелирования станет проходить выше нее.

Данная работа открывает серию исследований природы устойчивости образцов к повреждающим полевым воздействиям, выявлению возможной связи этого свойства со структурой потенциального барьера в изолирующем слое и другими характеристиками кремниевых МОП структур со сверхтонким окислом. Следующим шагом является построение на основе развитой в [14] методики реального потенциального рельефа, разделяющего полупроводник и полевой электрод. Решение этого вопроса даст ответ о справедливости сделанного выше предположения о форме изолирующего барьера, позволяющего объяснить асимметрию наблюдаемых туннельных ВАХ.

Работа выполнена при частичной финансовой поддержке гранта РФФИ № 16-07-00666 и Программы фундаментальных исследований президиума РАН № 32 „Наноструктуры: физика, химия, биология, основы технологийсб.

\section{Список литературы}

[1] A. Padovani, D.Z. Gao, A.L. Shluger, L. Larcher. J. Appl. Phys., 121, 155101 (2017).

[2] J.S. Suehle. In: Defects in Microelectronic Materials and Devices, ed. by D.M. Fleetwood, S.T. Pantelides and R.D. Schrimpf (CRC Press, 2008) chap. 15, p. 437. 
[3] J. Suñé, E.Y. Wu. In: Defects in Microelectronic Materials and Devices, ed. by D.M. Fleetwood, S.T. Pantelides and R.D. Schrimpf (CRC Press, 2008) chap. 16, p. 465.

[4] J. Nissan-Cohen. Appl. Surf. Sci., 39 (1-4), 511 (1989).

[5] T.R. Oldham, F.B. Mc Lean, H.E. Boesch, J.M. Mc Carrity. Semicond. Sci. Technol., 4 (12), 986 (1989).

[6] M.L. Reed. Semicond. Sci. Technol., 4 (12), 980 (1989).

[7] Е.И. Гольдман, Н.Ф. Кухарская, В.Г. Нарышкина, Г.В. Чучева. ФТП, 45 (7), 974 (2011).

[8] Е.И. Гольдман, Н.Ф. Кухарская, С.А. Левашов, В.Г. Нарышкина, Г.В. Чучева. ФТП, 51 (9), 1185 (2017).

[9] Е.И. Гольдман, А.И. Левашова, С.А. Левашов, Г.В. Чучева. ФТП, 49 (4), 483 (2015).

[10] Е.И. Гольдман, Н.Ф. Кухарская, С.А. Левашов, Г.В. Чучева. ФТП, 53 (1), 46 (2019).

[11] Е.И. Гольдман, Ю.В. Гуляев, Г.В. Чучева. Радиотехника, 8, 58 (2015).

[12] Е.И. Гольдман, Ю.В. Гуляев, А.Г. Ждан, Г.В. Чучева. ФТП, 44 (8), 1050 (2010).

[13] А.П. Барабан, В.В. Булавинов, П.П. Коноров. Электроника слоев на кремнии (Л., изд-во ЛГУ, 1988).

[14] Е.И. Гольдман, А.Г. Ждан, Н.Ф. Кухарская, М.В. Черняев. ФТП, 42 (1), 94 (2008).

Редактор Л.В. Шаронова

\section{Features of characteristics of field-resistant silicon-ultrathin-oxide-polysilicon structures}

\section{E.I. Goldman, S.A. Levashov, G.V. Chucheva}

Fryazino Branch of Kotel'nikov Institute of Radioengineering and Electronics, Russian Academy of Sciences, 141190 Fryazino, Russia

Abstract Results of studies of current-voltage and capacityvoltage characteristics features of stable resistant to field damage silicon-ultrathin-oxide-polysilicon structures are presented. It turned out, that total recharging of localized electronic states and minority carriers, concentrated at the substrate-insulator interface, which occurs with a change in the field voltage, is an order of magnitude higher, than that of samples susceptible to damage after the field stress. The tunnel current-voltage characteristic has a substantially asymmetric appearance: the current, flowing from the field electrode to the silicon substrate, is several orders of magnitude less, than the current, flowing from the silicon to the polysilicon, with the same values of the external voltage, falling on the insulating layer. To explain this asymmetry, it is assumed, that in the transition layer from polysilicon to oxide the potential barrier, separating the semiconductor electrode and the substrate, has a height of the order of $1 \mathrm{eV}$ and, therefore, always hinders the electrotransport; for reverse flows, this barrier stops limiting conductivity once the tunneling level becomes higher than it. 